\title{
Rituximab for Refractory Rheumatoid Arthritis: A 24-Week Open-Label Prospective Study
}

\author{
Ling Yin Ho, Chi Chiu Mok*, Chi Hung To, Anselm Mak, Mei Yuk Cheung and Ka Lung Yu
}

Department of Medicine and Geriatrics, Tuen Mun Hospital, Hong Kong SAR, China

\begin{abstract}
Objectives: To study the efficacy of rituximab in active rheumatoid arthritis (RA) patients refractory to disease modifying anti-rheumatic drugs (DMARDs) including the tumor necrosis factor (TNF)- $\alpha$ antagonists.

Methods: Adult patients with active RA despite adequate therapies with conventional DMARDs or anti-TNF $\alpha$ agents for at least 3 months were recruited. Inclusion criteria were: (1) Positive RF / anti-CCP; (2) $\geq 6$ swollen joints and $\geq 8$ tender joints; (3) ESR $\geq 28 \mathrm{~mm} / \mathrm{hr}$ or CRP $\geq 10 \mathrm{mg} / \mathrm{L}$. Eligible patients were given intravenous rituximab infusions at a dose of $1000 \mathrm{mg}$ on days 1 and 15 . Assessment was performed 4-weekly thereafter and included tender joint counts (TJC), swollen joint counts (SJC), physician's and patient's global assessment, patient's pain assessment (VAS 0-100 mm), disability index (HAQ-DI), quality of life (SF36), fatigue score (FACIT-F), ESR and CRP. The DAS28, EULAR and ACR responses at week 24 were evaluated.

Results: 10 patients (8 women and 2 men) were studied (mean age: 49 years; mean RA duration 7.4 years). Baseline TJC and SJC were $25.1 \pm 13.2$ and $12.8 \pm 5.4$ respectively. The mean DAS28 score was $7.1 \pm 0.7$, and the mean CRP and ESR levels were $52.3 \pm 60 \mathrm{mg} / \mathrm{L}$ and $95.8 \pm 32 \mathrm{~mm} / \mathrm{hr}$, respectively. The median number of failed DMARDs was 4 and two patients had failed anti-TNF $\alpha$ treatment. At week 24, there was a significant drop in TJC, SJC, ESR and CRP. The HAQ-DI score also decreased from 2.1 to $1.7(\mathrm{p}=0.04)$ while the total SF-36 score improved from 24.8 to 38.3 ( $\mathrm{p}=0.008$ ). Sixty percent of patients achieved EULAR moderate-to-good response. Half of the patients achieved ACR20 and two achieved ACR50 / 70 response. Only one patient experienced a minor infusion reaction.

Conclusions: Rituximab is effective and well tolerated in patients with refractory RA.
\end{abstract}

Keywords: Biologics, rituximab, anti-TNF $\alpha$, refractory, treatment.

\section{INTRODUCTION}

Rheumatoid arthritis (RA) is a chronic inflammatory disease that leads to significant disability and mortality. Conventional disease modifying anti-rheumatic drugs (DMARDs) include methotrexate (MTX), leflunomide (LEF), sulphasalazine (SSz), hydroxychloroquine (HCQ) and gold compounds. Despite the use of these agents as monotherapy or combination therapy, a substantial proportion of patients still cannot achieve a clinically meaningful clinical response. For patients with inadequate response to conventional DMARD therapies, the anti-tumor necrosis factor (TNF)- $\alpha$ agents such as infliximab, etanercept and adalimumab are treatment options. However, data from western countries have shown that around $50-60 \%$ of patients may achieve an American College of Rheumatology (ACR) 20 response [1-3]. Other patients are either withdrawn from the TNF $\alpha$ inhibitors because of inefficacy or experiencing adverse effects.

Because of the lack of universal efficacy of the TNF $\alpha$ inhibitors, a number of non-TNF $\alpha$ biological agents have been developed. One of these, rituximab, is a chimeric monoclonal antibody directed against the CD20 antigen on the surface of B cells [4]. Administration of rituximab leads to selective depletion of the $\mathrm{CD} 20$ positive $\mathrm{B}$ cells from the

\footnotetext{
*Address correspondence to this author at the Department of Medicine, Tuen Mun Hospital, Tsing Chung Koon Road, New Territories, Hong Kong, SAR China; Tel: (852) 2468 5386; Fax: (852) 2456 9100;

E-mail: ccmok2005@yahoo.com
}

body, leaving the stem cells and plasma cells unaffected because CD20 is not expressed on these cells.

Rituximab was first reported to be useful in refractory RA [5]. A phase II randomized controlled trial has shown efficacy in RA patients refractory to MTX [6]. With the addition of rituximab, an ACR20 response could be achieved in $73 \%$ of the patients as compared to $38 \%$ when MTX alone was continued. An extended follow-up of these patients at 48 weeks showed that the ACR response was sustained in some patients, with significant improvement in physical function, as measured by the Health Assessment Questionnaire Disability Index (HAQ-DI) being evident at week 24 [7]. A further extended follow-up of these patients at 1 and 2 years showed that a significantly higher proportion of patients who received rituximab plus MTX had improvement in HAQ-DI to a greater extent than the minimally clinically significant difference than those who received MTX alone [8].

A more recent double-blind placebo-controlled clinical study (DANCER) confirmed that in RA patients who were refractory to conventional therapies and the TNF $\alpha$ inhibitors, rituximab treatment ( 2 doses of $1000 \mathrm{mg}$ or $500 \mathrm{mg}$ ) led to significantly higher rates of ACR responses and EULAR moderate / good responses when compared to placebo infusion [9]. Rituximab was safe and well tolerated. The frequency of infectious complications was similar between treatment and placebo groups. Reactions to the first infusion occurred in one-third of patients receiving $1000 \mathrm{mg}$ rituximab infusion and could be minimized by intravenous corticosteroid pre-medication. 
The experience of rituximab in RA is limited in Hong Kong. We conducted this open-label cohort study to evaluate the efficacy and tolerability of rituximab in our local Chinese patients with active RA who were refractory to DMARD and anti-TNF $\alpha$ therapies.

\section{PATIENTS AND METHODS}

Patients with active RA despite conventional DMARD or anti-TNF $\alpha$ therapies were recruited. The inclusion criteria were: (1) $\geq 18$ years of age; (2) 1987 ACR criteria for the classification of RA [10]; (3) Ability to give informed consent and comply with the protocol and assessment; (4) Positive for either rheumatoid factor (RF) or anti-CCP antibody; (5) Active RA despite therapies with conventional DMARDs or the anti-TNF agents for at least 3 months, as evidenced by $\geq 6$ swollen joints (66 joint count system) and $\geq 8$ tender joints (68 joint count system); (6) Raised erythrocyte sedimentation rate $(\mathrm{ESR})$ to $\geq 28 \mathrm{~mm} / \mathrm{hr}$ or $\mathrm{C}$-reactive protein $(\mathrm{CRP}) \geq 10 \mathrm{mg} / \mathrm{L}$; (7) Stable dose of conventional DMARDs for at least 8 weeks prior to study entry or washout for at least 4 weeks (except for methotrexate and leflunomide, which were to be continued); (9) Use of anti-TNF $\alpha$ agents (infliximab, etanercept or adalimumab) for at least 3 months and washout (etanercept for at least 2 weeks, infliximab or adalimumab for at least 8 weeks).

Exclusion criteria were: (1) Major surgery (including joint surgery) within 8 weeks prior to study entry; (2) Functional class IV as defined by the ACR classification of functional status in RA [11]; (3) Treatment with other investigational agents within 4 weeks of study entry (eg. anti-IL6, anti-CD4); (4) Treatment with gamma globulin, plasmapheresis or Prosorba column within six months of study entry; (5) Intra-articular steroid injection 6 weeks before study entry; (6) Immunization with a live/attenuated vaccine within 4 weeks prior to study entry; (7) History of severe allergic reaction to human, humanized or murine monoclonal antibodies; (8) Active current bacterial, viral, fungal, mycobacterial or other infections; (9) Chronic hepatitis B or hepatitis C carriers; (10) History of malignancies, including solid tumors and hematologic malignancies; (11) Pregnant women or lactating mothers.

This study was approved by the Research and Ethics committee of our hospital. Written consent was obtained from all participants.

\section{Protocol}

Patients were given rituximab $1000 \mathrm{mg}$ by intravenous infusion on Day 1 and Day 15. Routine pre-medication was not given. Intravenous hydrocortisone $(100 \mathrm{mg})$ and chlorpheniramine $(10 \mathrm{mg}$ ) were to be given if patients developed a reaction to the infusion. Methotrexate was to be continued throughout the study period, together with folic acid. Leflunomide was also to be continued. The use of non-steroidal antiinflammatory drugs (NSAIDs) was allowed. Other DMARDs were stopped for at least 4 weeks prior to study entry.

\section{Clinical Assessments on Follow-Up Visits}

Patients were followed up four-weekly for clinical response and side effects to rituximab infusion. The following was assessed: tender joint count ([TJC]; 0-68), swollen joint count ([SJC]; 0-66), patient's pain assessment, patient's global assessment and physician's global assessment as recorded by visual analog scale (VAS) 0-100 mm. Physical functioning and disability was assessed by Health Assessment Questionnaire-Disability Index (HAQ-DI). Quality of life was assessed by the short form (SF)-36 questionnaire (Hong Kong Chinese version) [12]. Fatigue was assessed by the Functional Assessment of Chronic Illness TherapyFatigue (FACIT-F) subscale [13]. The DAS28 scores were calculated at each clinic visit.

\section{Study End Points}

The primary end-point of the trial was the proportion of patients who met the ACR20 and EULAR response criteria.

\section{Adverse Events}

A checklist of possible adverse events related to rituximab was evaluated for participants at each clinic visit.

\section{Statistical Analyses}

Unless otherwise stated, values expressed were mean \pm standard deviation (SD). Continuous variables from baseline to various time intervals were compared by the nonparametric Wilcoxon signed rank test. Statistical significance was defined as a p value of less than 0.05 , two-tailed. Statistical analyses were performed by the SPSS program (version 11.5, Chicago IL, 2002).

\section{RESULTS}

Twelve patients were screened but two of them were not eligible for study entry because of inadequate number of swollen and tender joints. Ten patients (two men and eight women) were finally recruited. All of them were ethnic Chinese. The median age was 49 years (range 42 to 62). The demographic and baseline disease characteristics are shown in Table 1. At study entry, all patients had very active RA as evidenced by a large number of TJC $(25.1 \pm 13.2)$ and SJC $(12.8 \pm 5.4)$, elevated ESR $(95.8 \pm 32.3 \mathrm{~mm} / \mathrm{hr})$ and CRP $(52.3 \pm 53.5 \mathrm{mg} / \mathrm{L})$ levels. Seven patients had radiological erosions at baseline. The median number of ineffective DMARDs in these patients was four. Two patients had failed anti-TNF $\alpha$ treatment (infliximab in one and both etanercept and infliximab in another patient).

\section{Table 1. Clinical Characteristics of Patients at Study Entry}

\begin{tabular}{l|l|}
\hline Age, years (median, range) & $49,36-62$ \\
Women & $8(80 \%)$ \\
Duration of RA, years & $8,1-19$ \\
TJC, median (IQR) & $23(17.5)$ \\
SJC, median (IQR) & $11.5(6.25)$ \\
HAQ-DI, median (IQR) & $2.1(0.67)$ \\
FACIT-F, median (IQR) & $27.0(10.8)$ \\
DAS28, median (IQR) & $7.2(1.2)$ \\
CRP, median (IQR) (mg/L) & $33.1(71.6)$ \\
ESR, median (IQR) (mm/hr) & $108(43.5)$ \\
Anti-CCP titer, median (IQR) & $76(225)$ \\
No. of patients with baseline radiographic erosions & $7(70 \%)$ \\
Median number of failed DMARDs & 4 \\
No. of patients who failed anti-TNF $\alpha$ treatment & $2(20 \%)$ \\
\hline RA = rheumatoid arthritis; TJC = tender joint count; SJC = swollen joint count; HAQ = \\
Health assessment questionnaire; FACIT-F = Functional Assessment of Chronic Illness \\
Therapy-Fatique; DAS = disease activity score; CRP = C-reactive protein; ESR = \\
erythrocyte sedimentation rate; CCP = citrullinated cyclic peptide; DMARD = disease \\
modifying anti-rheumatic drugs; TNF = tumor necrosis factor.
\end{tabular}


Figs. $(\mathbf{1 , 2})$ show the number of patients who achieved the EULAR and ACR responses, respectively, at baseline and different time intervals. At week 24, 60\% of the patients achieved EULAR moderate-to-good response. Half of the patients achieved ACR20, one achieved ACR50 and one achieved ACR70 response. The mean DAS28 scores decreased significantly from $7.1 \pm 0.7$ at baseline to $5.6 \pm 1.2$ at week $24(\mathrm{p}=0.005)$. Of the two patients who had failed anti$\mathrm{TNF} \alpha$ treatment, one showed moderate EULAR response while the other did not meet the criteria for a clinical response despite a numerical improvement in the joint counts and DAS score.

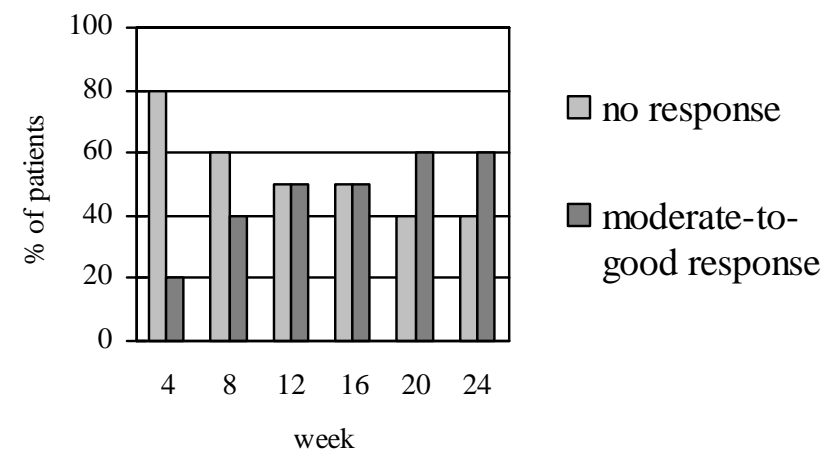

Fig. (1). EULAR responses at different time intervals following treatment with rituximab.

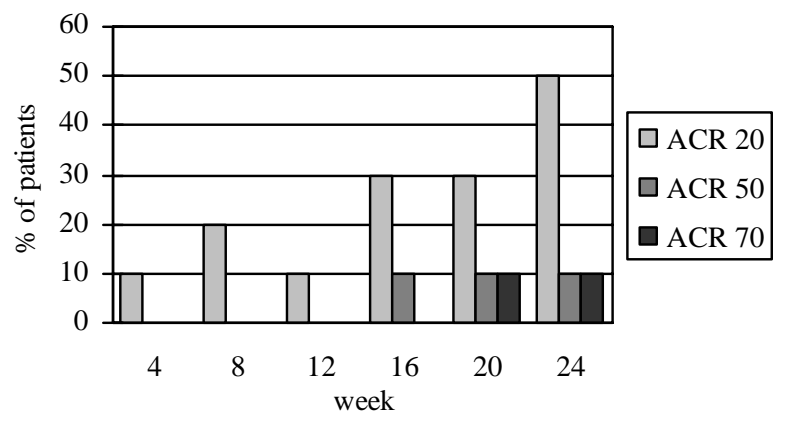

Fig. (2). ACR20, ACR50 and ACR70 responses at different time intervals following treatment with rituximab.

Comparing the data at week 24 with baseline, there was a significant drop in the mean number of TJC $(25.1 \pm 13.2$ to $13.9 \pm 14.7, \mathrm{p}=0.005)$, and $\mathrm{SJC}(12.8 \pm 5.4$ to $5.7 \pm 5.2$, $\mathrm{p}=0.02$ ). This was accompanied by a significant improvement in the mean levels of ESR $(95.8 \pm 32.2$ to $65.4 \pm 41$ $\mathrm{mm} / \mathrm{hr}, \mathrm{p}=0.01)$ and CRP $(52.3 \pm 53.5$ to $30.1 \pm 42.6 \mathrm{mg} / \mathrm{L}$, $\mathrm{p}=0.02)$.

The mean HAQ-DI score decreased from $2.1 \pm 0.4$ to 1.7 $\pm 2.6(\mathrm{p}=0.04)$. Seventy percent of patients had an improvement from baseline of greater than 0.25 points of the HAQDI, ie. the minimum clinically important difference (MCID) as described by Strand et al. [8]. Both the mental and physical health sub-scores of the SF-36 increased significantly from baseline to week 24 . The mean fatigue (FACIT-F) score also improved significantly from $27.7 \pm 7.4$ to $20.1 \pm$ $6.6(\mathrm{p}=0.007)$.
Finally, the mean titers of RF dropped significantly from baseline to week $24(42.0 \pm 14$ to $30.4 \pm 12 \mathrm{IU} / \mathrm{ml}$; $\mathrm{p}=0.005)$. The mean titers of anti-CCP also showed a trend of decrease (114 \pm 103 to $105 \pm 106$ units; $\mathrm{p}=0.09)$.

\section{Adverse Events}

Only one patient experienced transient urticaria during the first dose of rituximab infusion. It resolved after intravenous hydrocortisone and chlorpheniramine. Drug infusion was completed without further problem. Premedication with hydrocortisone and chlorpheniramine was given to this patient before the second infusion. A mild skin reaction was noted during the infusion. This patient was known to have history of aspirin allergy and had had inadequate response to either infliximab and etanercept. No other adverse events were reported in other patients throughout the study.

\section{DISCUSSION}

B cells may play an important role in the pathogenesis of RA. First, B cells may act as antigen presentation cells and activate $\mathrm{T}$ cells through the interaction between the MHC class II molecule and the T cell receptor. Second, B cells generate autoantibodies such as rheumatoid factor and anticitrullinated cyclic peptide (anti-CCP) antibodies. These antibodies may increase the inflammatory processes in the joints by promoting immune complex formation and complement activation, and hence lead to joint erosion and damage.

The efficacy of B cell depletion in rheumatoid arthritis confirms the role of B cells in the pathogenesis of RA $[6,9]$. The proportion of patients who achieved ACR responses after rituximab treatment is very similar to that of the antiTNF $\alpha$ agents [1-3]. In contrast to the TNF $\alpha$ inhibitors, infective complications including tuberculosis and opportunistic infections are much less common with rituximab.

Our study showed that rituximab was efficacious for patients with active RA refractory to multiple DMARDs. Despite the lack of a placebo group, the improvement in our patients at the end of the study was unlikely to be spontaneous because they had active RA for at least 3 months prior to study entry. The ACR response rates achieved at week 24 were quite similar to those reported in a placebo-controlled trial of rituximab in RA [9]. In addition to the improvement in joint swelling and tenderness, there was also a significant drop in the levels of serum inflammatory markers and RF titers, which was coupled with a significant improvement in quality of life and the disability index.

Despite the fact that we did not routinely prescribe intravenous or oral corticosteroids as pre-medication before rituximab infusion, none or our patients experienced serious infusion reactions. Only one patient developed mild infusion reaction which was settled with intravenous hydrocortisone injection. Rituximab infusion was not interrupted. No infection, mild or serious, was reported throughout the 24-week follow-up in our patients. Thus, the safety profile is quite similar to that in controlled trials $[9,14]$ in which no significant difference in the frequency of adverse events could be demonstrated between rituximab and placebo groups of patients.

Apart from MTX and other conventional DMARDs, rituximab may also be effective in RA patients who do not 
respond to the anti-TNF $\alpha$ agents. One of our patients who had failed infliximab achieved EULAR moderate response after one single course of rituximab. An open-label uncontrolled study of rituximab (100 mg on week $1,375 \mathrm{mg} / \mathrm{m} 2$ on week $2,500 \mathrm{mg} / \mathrm{m} 2$ on week 3 and 4) in 13 RA patients, 6 of whom were refractory to anti-TNF $\alpha$, showed that two third of patients achieved the ACR20 response at week 28 [15]. Another small uncontrolled series involving 10 patients showed that rituximab treatment $(1000 \mathrm{mg}$ for 2 doses 2 week apart) resulted in moderate / good DAS28 response in $80 \%$ of patients [16]. A more recent randomized controlled study called REFLEX trial (Randomized Evaluation of Long-Term Efficacy of Rituximab in RA) demonstrated that in patients who had inadequate response to one or more antiTNF $\alpha$ agents, 2 infusions of $1000 \mathrm{mg}$ of rituximab in addition to background MTX led to significantly higher response rates than MTX alone (EULAR moderate-to-good response $65 \%$ vs $22 \%$ ) [14].

In summary, the current study showed that rituximab is effective in our local Chinese patients with persistently active RA despite multiple DMARD therapies including the anti-TNF $\alpha$ agents. Rituximab is a relatively safe option to be considered in these patients.

\section{ACKNOWLEDGEMENTS}

This was an investigator-initiated study. Rituximab samples were provided by Roche.

\section{REFERENCES}

[1] St Clair EW, van der Heijde DM, Smolen JS, et al. Combination of infliximab and methotrexate therapy for early rheumatoid arthritis: a randomized, controlled trial. Arthritis Rheum 2004; 50: 3432-43.

[2] Bathon JM, Martin RW, Fleischmann RM, et al. A comparison of etanercept and methotrexate in patients with early rheumatoid arthritis. N Engl J Med 2000; 343: 1586-93.

[3] Breedveld FC, Weisman MH, Kavanaugh AF, et al. The PREMIER study: A multicenter, randomized, double-blind clinical trial of combination therapy with adalimumab plus methotrexate versus methotrexate alone or adalimumab alone in patients with early, aggressive rheumatoid arthritis who had not had previous methotrexate treatment. Arthritis Rheum 2006; 54: 26-37.
[4] Edwards JC, Leandro MJ, Cambridge G. B lymphocyte depletion therapy with rituximab in rheumatoid arthritis. Rheum Dis Clin North Am 2004; 30: 393-403, viii.

[5] Cambridge G, Leandro MJ, Edwards JC, et al. Serologic changes following B lymphocyte depletion therapy for rheumatoid arthritis. Arthritis Rheum 2003; 48: 2146-54.

[6] Edwards JC, Szczepanski L, Szechinski J, et al. Efficacy of B-celltargeted therapy with rituximab in patients with rheumatoid arthritis. N Engl J Med 2004; 350: 2572-81.

[7] Pavelka K, Nahir AM, Edwards JC, et al. Improvement in patientreported outcomes with rituximab in patients with rheumatoid arthritis. Ann Rheum Dis 2004; 50(Suppl 1): 289-90.

[8] Strand V, Balbir-Gurman A, Pavelka K, et al. Sustained benefit in rheumatoid arthritis following one course of rituximab: improvements in physical function over 2 years. Rheumatology 2006; 45: 1505-1513.

[9] Emery P, Fleischmann RM, Filipowicz-Sosnowska A, et al., for the DANCER Study Group. The efficacy and safety of rituximab in patients with active rheumatoid arthritis despite methotrexate treatment. Results of a phase IIb randomized, double-blind, placebocontrolled, dose-ranging trial. Arthritis Rheum 2006; 54: 13901400.

[10] Arnett FC, Edworthy SM, Bloch DA, et al. The American Rheumatism Association 1987 revised criteria for the classification of rheumatoid arthritis. Arthritis Rheum 1988; 31: 315-24.

[11] Hochberg M, Chang RW, Dwosh I, Lindsey S, Pincus T, Wolfe F. The American College of Rheumatology 1991 revised criteria for the classification of global functional status in rheumatoid arthritis. Arthritis Rheum 1992; 35: 498-502

[12] Lam CL, Tse EY, Gandek B, Fong DY. The SF-36 summary scales were valid, reliable, and equivalent in a Chinese population. J Clin Epidemiol 2005; 58: 815-22.

[13] Cella D, Yount S, Sorensen M, et al. Validation of the Functional Assessment of Chronic Illness Therapy Fatigue Scale relative to other instrumentation inpatients with rheumatoid arthritis. Arthritis Rheum 2005; 32: 811-9.

[14] Cohen SB, Emery P, Greenwald MW, et al. for the REFLEX Trial Group. Rituximab for rheumatoid arthritis refractory to anti-tumor necrosis factor therapy. Results of a multicenter, randomized, double-blind, placebo-controlled, phase III trial evaluating primary efficacy and safety at twenty-four weeks. Arthritis Rheum 2006; 54: 2793-2806

[15] Higashida J, Wun T, Schmidt S, et al. Safety and efficacy of rituximab in patients with rheumatoid arthritis refractory to disease modifying anti-rheumatic drugs and anti-tumor necrosis factor-a treatment. J Rheumatol 2005; 32: 2109-15.

[16] Brulhart L, Ciurea A, Finckh A, et al. Efficacy of B cell depletion in patients with rheumatoid arthritis refractory to anti-tumour necrosis factor a agents: an open-label observational study. Ann Rheum Dis 2006; 65: 1255-1257. 